\title{
Predicting the effects of radiotherapy based on diffusion kurtosis imaging in a xenograft mouse model of esophageal carcinoma
}

\author{
AN-DU ZHANG ${ }^{1 *}$, XIAO-HUA SU ${ }^{2 *}$, YAN-FEI WANG ${ }^{3}$, GAO-FENG SHI ${ }^{3}$, CHUN HAN $^{1}$ and NAN ZHANG ${ }^{1}$ \\ ${ }^{1}$ Department of Radiotherapy, Hebei Medical University Fourth Affiliated Hospital/Hebei Provincial Tumor Hospital; \\ ${ }^{2}$ Department of Oncology, Hebei General Hospital; ${ }^{3}$ Department of CT and MRI, Hebei Medical University \\ Fourth Affiliated Hospital/Hebei Provincial Tumor Hospital, Shijiazhuang, Hebei 050011, P.R. China
}

Received May 9, 2020; Accepted November 20, 2020

DOI: $10.3892 /$ etm.2021.9758

\begin{abstract}
The aim of the present study was to assess the predictive value of diffusion kurtosis imaging (DKI) on the effects of radiotherapy in a xenograft model of esophageal cancer. A total of 40 tumor-bearing mice, established by injection of Eca-109 cells in nude mice, were used. The experimental group $(n=24)$ received a single dose of $15 \mathrm{~Gy}$ (6 MV by X-ray), and the control group $(n=16)$ did not receive any treatment. Tumor volume, apparent diffusion coefficient (ADC), mean kurtosis (MK) and mean diffusivity (MD) of the two groups were compared, and the expression of aquaporin (AQP) 3 and necrosis ratio at matched time points in xenografts were also observed. There was a significant difference between the two groups from the 7th day of radiotherapy onwards; the xenograft volume of the experimental group was significantly smaller compared with the control group $(\mathrm{P}<0.05)$. On the 3rd day, the ADC and MD of the experimental group was significantly higher compared with the control group, and MK was significantly lower compared with the control group $(\mathrm{P}<0.05)$. On the 3rd day, AQP3 expression in the experimental group was lower compared with the control group, and the proportion of necrotic cells was higher compared with the control group $(\mathrm{P}<0.05)$. Single large fraction dose radiotherapy inhibited the growth of a xenografted esophageal tumor. Changes in ADC, MK and MD were observed prior to morphological changes in the tumor. The change in AQP3 expression and necrosis ratio was in also agreement with the DKI parameters assessed. DKI may thus
\end{abstract}

Correspondence to: Professor Gao-Feng Shi, Department of CT and MRI, Hebei Medical University Fourth Affiliated Hospital/Hebei Provincial Tumor Hospital, 12 Jiankang Road, Shijiazhuang, Hebei 050011, P.R. China

E-mail: gaofengs62@163.com

*Contributed equally

Key words: diffusion kurtosis imaging, mean kurtosis, mean diffusivity, apparent diffusion coefficient, radiotherapy provide early predictive ability on the effect of radiotherapy in esophageal carcinoma.

\section{Introduction}

Diffusion-weighted magnetic resonance imaging (DWMRI) is a functional imaging technique based on the microscopic random translational motion of water molecules in biological tissues (1). The magnitude of this motion is characterized by its apparent diffusion coefficient (ADC) values. Water molecules can diffuse relatively freely in tissues with normal cellularity, which results in a loss of signal on diffusion-weighted imaging (DWI) and thus a high ADC (1). Conversely, the diffusion of water is restricted in tissues with increased cellularity (such as tumors), resulting in a high signal on DWI and a low ADC (2). DWMRI holds promise for use as a method of detecting and diagnosing cancer due to its sensitivity to macromolecular and microstructural changes that occur at the cellular level and prior to anatomical changes (3). ADC in DWI can be used predict the prognosis of patients with esophageal squamous cell carcinoma and treatment response (3). Traditionally, determination of the ADC is based on the assumption of a Gaussian distribution of displacement probabilities of water molecules due to water self-diffusion $(4,5)$. The DWI approach to data analysis was founded on the hypothesis that water molecules diffuse within a voxel following a single direction with Gaussian behavior and no restrictions $(4,5)$. The discovery of aquaporin (AQP) by Agre et al (6) in 1993 challenged this concept. AQPs are a family of 13 small hydrophobic integral transmembrane water channel proteins involved in transcellular and transepithelial water movement, transport of fluid and cell migration $(7,8)$. Due to the presence of AQPs, water molecules within biological tissues exhibit a non-Gaussian phenomenon known as diffusion kurtosis imaging (DKI), as proposed by Jensen and Helpern and Jensen et al (4,9). This approach evaluates the kurtosis coefficient $(\mathrm{K})$, which shows the deviation of diffusion from the Gaussian-predicted approach, and the diffusion coefficient (D) corrected for non-Gaussian bias. Several studies have shown that DKI is more accurate than traditional ADC mapping in tumor detection and grading assessment $(5,10-15)$. DWI using quantitative parameters (ADC and 
DKI) may serve as an imaging biomarker to more effectively identify patients that are more likely to benefit from aggressive neoadjuvant treatments $(16,17)$. In the present study, an animal model of esophageal carcinoma was used to examine the predictive value of DKI on the effects of radiotherapy, and the associated mechanisms underlying the clinical applications of the technology were investigated.

\section{Materials and methods}

Cell culture. Eca-109 human esophageal carcinoma cells (Shanghai Institute of Biological Sciences) were cultured at $37^{\circ} \mathrm{C}$ in a $5 \% \mathrm{CO}_{2}$ incubator with RPMI-1640 medium (Costar; Corning, Inc.) supplemented with 10\% FBS (Gibco; Thermo Fisher Scientific, Inc.). Adherent cell cultures were used to increase growth during passaging.

Experimental animals and model construction. Immunedeficient BALB/c nude mice (4-6 weeks old; male, 18-20 g) were purchased from Beijing Vital River Laboratory Animal Technology Co., Ltd.; Charles River Laboratories, Inc., and reared in the Animal Experiment Center of Hebei Medical University Fourth Affiliated Hospital and Hebei Provincial Tumor Hospital in a specific pathogen-free environment with a $12 \mathrm{~h}$ light/dark cycle, ad libitum access to water and food, $50 \pm 10 \%$ relative humidity and $23 \pm 2^{\circ} \mathrm{C}$ temperature. The cell suspension was amplified by the pancreatic digestion enzyme method. Using $1 \mathrm{ml}$ injection to extract the collected cell suspensions, the right forelimbs of nude mice were chosen as the inoculation site, and the number of inoculated cells in every nude mice was $5 \times 10^{6} / 0.2 \mathrm{ml}$. At $\sim 2$ weeks after inoculation, a short diameter of about $10 \mathrm{~mm}$ subcutaneous tumor can be formed.

Nude mice were anesthetized using $2 \%$ pentobarbital injections of $0.05 \mathrm{ml} / \mathrm{mouse}(50 \mathrm{mg} / \mathrm{kg}$ ) before MRI scanning. Animal health and behavior were monitored once every other day, the cell injections produced no observable behavioral effects and these mice gained weight normally. A total of 132 mice were used, and eight mice were found dead caused by overdose of pentobarbital at the beginning of the experiment. At the end of the experiments, all animals were euthanized by overdose of pentobarbital for histological experiments. The mice stopped breathing and their heartbeats stopped. The entire experiment lasted 42 weeks. All animal experiments were approved by the Animal Protection Committee of the Hebei Medical University Fourth Affiliated Hospital and Hebei Provincial Tumor Hospital (approval no. 201618; Hebei, China).

Experimental grouping. The schematic diagram of experimental groupings is shown in Fig. 1. For experiment 1, a total of 40 tumor-bearing nude mice were randomly divided into two groups. The experimental group (24 mice) received $6 \mathrm{MV}-\mathrm{X}$ ray of $15 \mathrm{~Gy}$ radiotherapy (18). The control group (16 mice) did not receive treatment. The two groups were scanned before radiotherapy once every other day, for 1 month after radiotherapy, for a total of 16 time points.

For experiment 2, according to the results of the initial radiotherapy experiments, seven key time points were selected, and mice were divided into seven groups, and 12 mice in each group were randomly divided into the experimental and control groups.
MRI scans were performed on the mice before radiotherapy and $1,3,5,7,17$ and 29 days after radiotherapy. After completing the MRI scan, the tumor-bearing nude mice were immediately sacrificed via dislocation of the cervical vertebra, and the tumor tissue was removed and soaked in $10 \%$ formalin solution.

Irradiation mode. An Elekta linear accelerator (Elekta Instrument $\mathrm{AB}$ ) was utilized with a $6 \mathrm{MV}$-X ray. The shooting field was $2 \times 2 \mathrm{~cm}$. The source skin distance was $100 \mathrm{~cm}$. The dose rate was $300 \mathrm{cGy} / \mathrm{min}$, and a single dose of $15 \mathrm{~Gy}$ was administered and covered with $1 \mathrm{~cm}$ tissue compensation membrane. All the tumor-bearing nude mice in the experimental group were fixed in the prone position and received radiotherapy whilst they were awake.

MRI examination. MRI examination involved a Siemens 3.0 T MRI scanner (Siemens Healthineers), loop coil and scanning sequence, including T1 weighted imaging (T1WI), T2 weighted imaging (T2WI) and DWI sequences (Table I), with a diffusion time of $\sim 10 \mathrm{~min}$. B (dispersion-sensitive gradient) selection was as follows $(19,20): 0,500,800,1,000$, $1,500,2,000,2,500$ and $3,000 \mathrm{sec} / \mathrm{mm}^{2}$. Nude mice were anesthetized using $2 \%$ pentobarbital injection $(0.05 \mathrm{ml} / \mathrm{mouse})$ before scanning, and the tumor-bearing nude mice were wrapped in fresh pork to reduce the magnetic susceptibility artifact (21) and placed in the loop coils.

Calculation of parameters and measurement of tumor volume. The DKI parametric maps were reconstructed using a prototype software (Body Diffusion Toolbox 1.3; Siemens Healthineers). Regions of interest (ROI) was delineated on special $\mathrm{b}$ value images $\left(1,000 \mathrm{~s} / \mathrm{mm}^{2}\right)$ due to a high contrast between lesions and background. All ROIs were drawn with a criteria where as much lesion tissue as possible were included, and as little unconcerned tissue as possible were included. If necessary, a T1WI contrast-enhanced or T2WI images were referred to. For the measurement of volume or parameters, ITK SNAP software version 3.8.0 (https://itk.org/) was used, which is an open source image analysis tool that can be used in medical image segmentation and measurement. Mean values of measured lesions on concerned parametric maps were used as the final index. MR images across all timepoints were analyzed by the consensus of two experienced radiologists (with 10 and 8 years of experience in clinical MR, respectively). To assess the intra-observer reproducibility of the measured values, consistency analysis was performed on the 50 images using the values measured by both the radiologists, and the averages of the values measured by the two radiologists were used for final analysis (Fig. 2).

Detection of $A Q P$ expression in tumors. Total RNA was extracted from the frozen tumors tissue samples using TRIzol $^{\circledR}$ reagent (Invitrogen; Thermo Fisher Scientific, Inc.). RNA quality was assessed using a NanoDrop 1000 (Thermo Fisher Scientific, Inc.). cDNA was synthesized using the First-strand cDNA synthesis kit (Thermo Fisher Scientific, Inc.) according to the manufacturer's instructions. Reverse transcription-quantitative PCR (RT-qPCR) was performed on an ABI PRISM 7500 (Applied Biosystems) using SYBR Green Supermix with ROX (Invitrogen; Thermo Fisher 
A

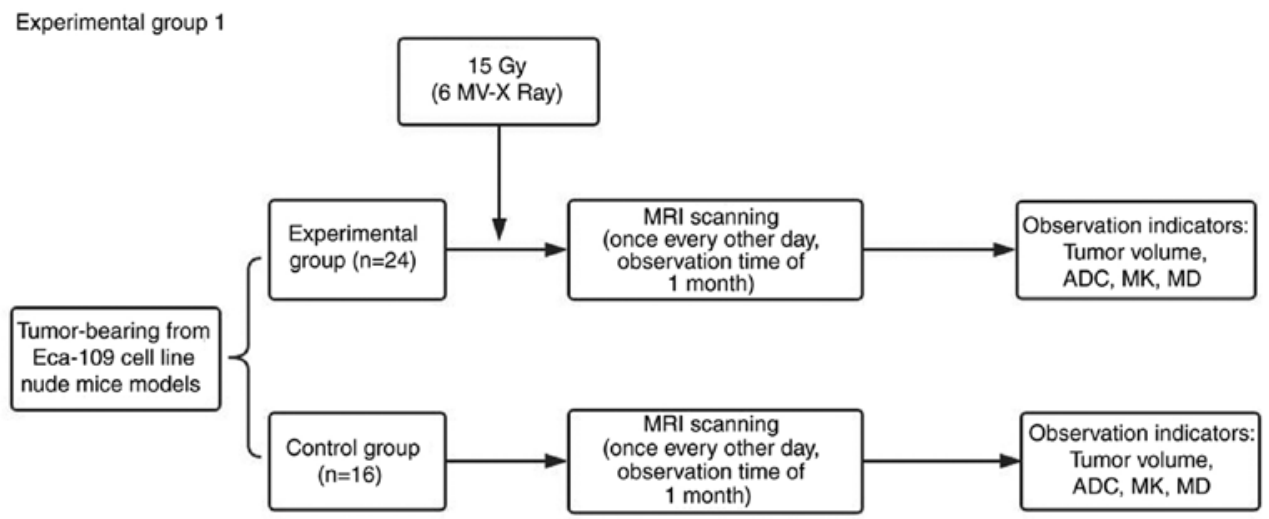

B

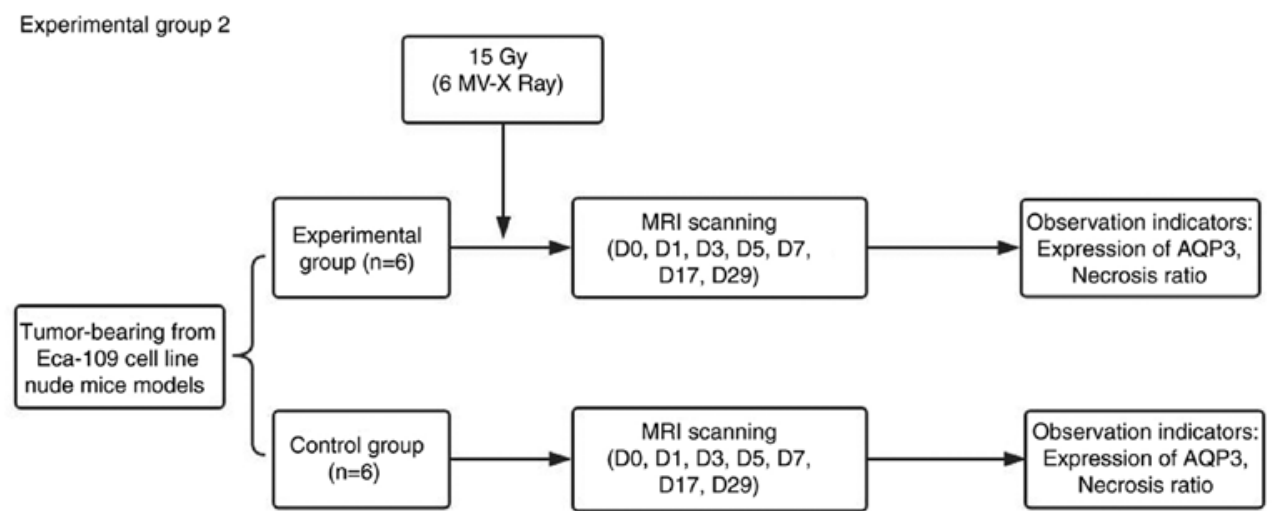

Figure 1. Experimental flow chart. (A) Flow chart of experimental grouping 1. (B) Flow chart of experimental grouping 2. ADC, apparent diffusion coefficient; MK, mean kurtosis; MD, mean diffusivity; AQP3, aquaporin 3; D, day.

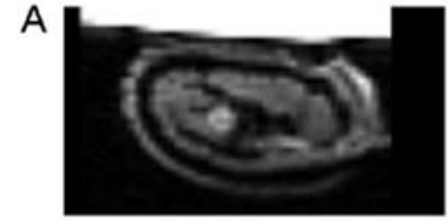

Day 0 (control group)

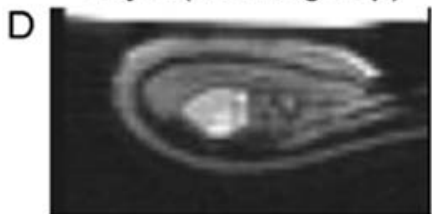

Day 29 (control group)

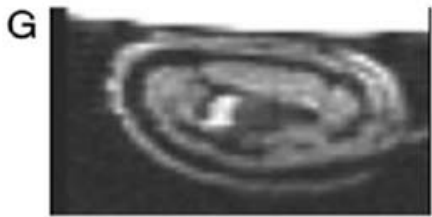

Day 0 (experimental group)

J

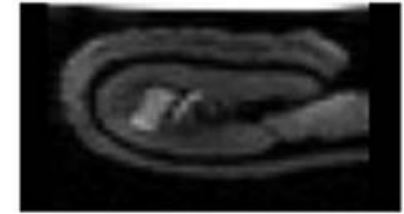

Day 29 (experimental group)
B

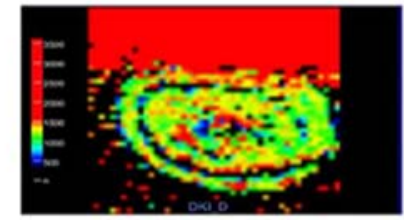

Day 0 (control group)

E

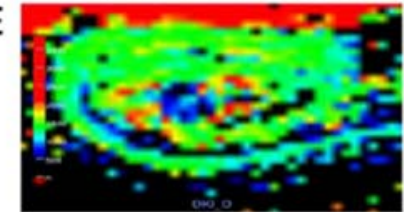

Day 29 (control group)

$\mathrm{H}$

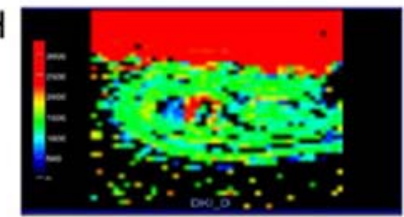

Day 0 (experimental group)

$\mathrm{K}$

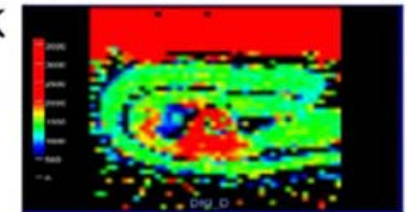

Day 29 (experimental group)

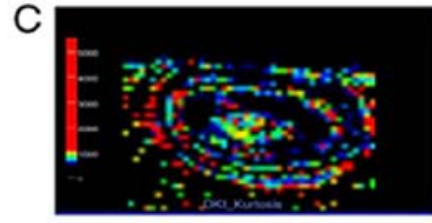

Day 0 (control group)

F

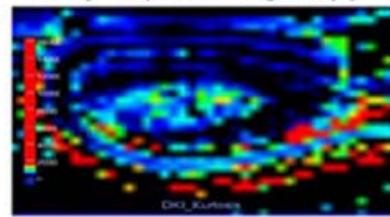

Day 29 (control group)

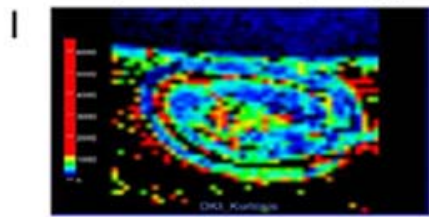

Day 0 (experimental group)

L

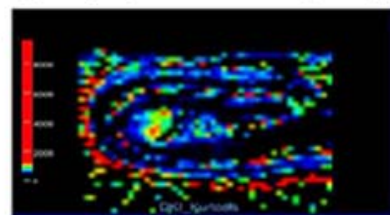

Day 29 (experimental group)

Figure 2. MRI scan image. (A) ADC, (B) MD and (C) MK maps of tumors on D0 in the control group. (D) ADC, (E) MD and (F) MK maps of tumors on D29 in the control group. (G) ADC, (H) MD and (I) MK maps of tumors on D0 in the experimental group. (J) ADC, (K) MD and (L) MK maps of tumors on D29 in the experimental group. ADC, apparent diffusion coefficient; MK, mean kurtosis; MD, mean diffusivity; D, day. 
Table I. MRI scan sequences and parameters.

\begin{tabular}{lrrr}
\hline Scan parameters & $\begin{array}{c}\text { T1WI } \\
\text { (Location) }\end{array}$ & $\begin{array}{c}\text { T2WI } \\
\text { (Coronal) }\end{array}$ & $\begin{array}{c}\text { DWI } \\
\text { (Axial) }\end{array}$ \\
\hline TR (msec) & 700 & 3,500 & 2,400 \\
TE (msec) & 29 & 81 & 76 \\
TA & $2: 00$ & $2: 08$ & $13: 26$ \\
FOV read (mm) & 90 & 180 & 100 \\
FOV phase (\%) & 100 & 100 & 100 \\
Slice thickness (mm) & 2.5 & 1.4 & 3.0 \\
Number of excitations & 1 & 1 & 3 \\
Dist. factor $(\%)$ & 30 & 30 & 40 \\
Flip angle $\left({ }^{\circ}\right)$ & 150 & 90 & 90 \\
Base resolution & 448 & 320 & 34 \\
Phase resolution & 336 & 256 & 34 \\
Scan trace & - & - & 3 \\
\hline
\end{tabular}

T1WI, T1 weighted imaging; T2WI, T2 weighted imaging; TR, repetition time; TE, echo time; TA, average time; FOV, field of view; DWI, diffusion-weighted imaging; Averages, number of excitation; Dist. factor, the ratio of the distance and thickness between the two layers.

Scientific, Inc.) according to the manufacturer's instructions. Thermocycling conditions were as follows: $10 \mathrm{~min}$ at $95^{\circ} \mathrm{C}$, followed by $10 \mathrm{sec}$ at $95^{\circ} \mathrm{C}, 30 \mathrm{sec}$ at $58^{\circ} \mathrm{C}$ and $25 \mathrm{sec}$ at $72^{\circ} \mathrm{C}$, for a total of 40 cycles, then $1 \mathrm{~min}$ at $95^{\circ} \mathrm{C}, 30 \mathrm{sec}$ at $72^{\circ} \mathrm{C}$ and $30 \mathrm{sec}$ at $95^{\circ} \mathrm{C}$. Primers for AQPs were purchased from GeneCopoeia, Inc. (Product IDs: GAPDH, HQP006940; AQP1, HQP009689; AQP2, HQP009705; AQP3, HQP009724; AQP4, HQP009734; AQP5, HQP101117; AQP6, HQP009760; AQP7, HQP009768; AQP8, HQP009420; AQP9, HQP009790; AQP10, HQP021803; AQP11, HQP099040 and AQP12B, HQP068062). Amplification signals for samples were normalized to GAPDH, and the relative expression was calculated using the $2^{-\Delta \Delta \mathrm{Cq}}$ method (22).

Western blotting analysis. Total protein from xenograft tumor was extracted using RIPA lysis buffer with freshly added protease inhibitor (Roche Diagnostics). The expression of AQP3 protein in tumors was detected using western blotting. Briefly, $60 \mu \mathrm{g}$ total protein extract was separated by $10 \%$ SDS-PAGE followed by transfer onto a PVDF membrane (Roche Diagnostics), which was determined using a BCA kit (Sigma-Aldrich; Merck KGaA). Membranes were blocked with $5 \%$ skim milk at $25^{\circ} \mathrm{C}$ for $2 \mathrm{~h}$, incubated with rabbit polyclonal antibodies against human AQPs (1:500; cat. no. ARG10648; Arigo Biolaboratories Corp.) or GAPDH (1:10,000; Santa Cruz Biotechnology, Inc.) overnight at $4^{\circ} \mathrm{C}$, followed by incubation with secondary Goat anti-Rabbit IgG antibodies (HRP conjugated; 1:5,000; cat. no. ARG65351; Arigo Biolaboratories Corp.) at $25^{\circ} \mathrm{C}$ for $2 \mathrm{~h}$. Proteins were visualized with an ECL reagent (Thermo Fisher Scientific, Inc.) using the FluorChem ${ }^{\circledR}$ HD2 protein imprinting imaging system (Alpha InnoTec).

Detection of necrotic ratio in tumors. The pathological sections of tumors obtained from the xenograft mouse model of esophageal carcinoma were fixed in $10 \%$ neutral buffered formalin solution at $25^{\circ} \mathrm{C}$ for $24 \mathrm{~h}$. Tumor tissues were paraffin embedded and cut into $4-\mu \mathrm{m}$ thick sections. The sections were stained with Hematoxylin and Eosin, and were scored by two experienced hepatopathologist (YW and NZ) in a blinded manner. The necrotic ratio was based on three randomly selected complete and non-overlapping macroscopic views (light microscope; Nikon Corporation; magnification, $\mathrm{x} 40$ ) for each tissue slice, and analyzed using ImageJ (v2.1.4.7, National Institutes of Health). The following equation was used: Tumor necrosis ratio $=($ necrosis area/tumor area $) \times 100 \%$.

Statistical analysis. Statistical analysis was performed using SPSS version 22.0 (IBM Corp.). The normality of quantitative data was assessed using a Kolmogorov-Smirnov test. All of data conformed to the normal distribution, and are presented as the mean $\pm \mathrm{SD}$. Continuous data that were normally distributed were analyzed using a Student's t-test. The variables at different time points were compared using repeated measures ANOVA. $\mathrm{P}<0.05$ was considered to indicate a statistically significant difference.

\section{Results}

Comparison of tumor volume between the experimental and control groups before and after radiotherapy. In experiment 1, the growth doubling time was 17 days in the experimental group and 5 days in the control group. There was a significant difference in tumor volume in the experimental group at different time points $(\mathrm{F}=39.366 ; \mathrm{P}<0.001)$, and tumor volume in the control group at different time points $(\mathrm{F}=61.561$; $\mathrm{P}<0.001)$. The volume, $\mathrm{V}_{0}$, of the two groups was $1.043 \pm 0.374$ and $0.917 \pm 0.310 \mathrm{~cm}^{3}$, respectively, and there was no statistically significant difference between the two groups $(\mathrm{P}=0.270)$. The tumor volume in the control group was approximately equal to the experimental group on the 1st and 3rd days, and significant differences between the two were observed on the 7th day after radiotherapy, and the xenograft volume of the experimental group was significantly smaller compared with the control group $(\mathrm{P}<0.05$; Table II).

Comparison of $A D C$ before and after radiotherapy. The ADC of the experimental group decreased on the 1st day and increased rapidly on the 3rd day peaking on the 7th day, after which, ADC remained relatively stable and gradually declined after 17 days. However, the ADC in the control group began to decrease from the 1st day and showed a monotonical decrease which continued at a relatively low level from the 7th day. There were differences in ADC in the experimental group at different time points $(\mathrm{F}=12.613 ; \mathrm{P}<0.001)$, and there were significant differences in $\mathrm{ADC}$ in the control group at different time points $(\mathrm{F}=3.288 ; \mathrm{P}=0.009)$. There was no significant difference between the two groups in $\mathrm{ADC} 0$ and ADC1 $(\mathrm{P}>0.05)$. A significant difference between ADC in the two groups was first observed on day 3 after radiotherapy, where the experimental group showed a significantly higher ADC than the control group $(\mathrm{P}<0.05$; Table III).

Comparison of $M K$ before and after radiotherapy. The MK of the experimental group began to decline on the 1st 
Table II. Comparison of transplanted tumor volumes between experimental and control groups after radiotherapy.

\begin{tabular}{lllr}
\hline Day & $\begin{array}{c}\text { Control } \\
\text { group }\left(\mathrm{cm}^{3}\right)\end{array}$ & $\begin{array}{c}\text { Experimental } \\
\text { group }\left(\mathrm{cm}^{3}\right)\end{array}$ & P-value \\
\hline D0 & $0.917 \pm 0.310$ & $1.043 \pm 0.374$ & 0.270 \\
D1 & $1.116 \pm 0.389$ & $1.275 \pm 0.460$ & 0.264 \\
D3 & $1.610 \pm 0.619$ & $1.638 \pm 0.745$ & 0.903 \\
D5 & $2.098 \pm 0.815$ & $1.794 \pm 0.773$ & 0.240 \\
D7 & $2.671 \pm 0.915$ & $1.729 \pm 0.906$ & 0.003 \\
D9 & $3.267 \pm 1.240$ & $1.872 \pm 0.918$ & $<0.001$ \\
D11 & $3.554 \pm 1.428$ & $1.890 \pm 0.928$ & $<0.001$ \\
D13 & $4.107 \pm 1.820$ & $1.968 \pm 0.972$ & $<0.001$ \\
D15 & $4.803 \pm 2.207$ & $2.030 \pm 0.997$ & $<0.001$ \\
D17 & $5.339 \pm 2.426$ & $2.101 \pm 1.082$ & $<0.001$ \\
D19 & $5.919 \pm 2.662$ & $2.118 \pm 1.068$ & $<0.001$ \\
D21 & $6.473 \pm 2.726$ & $2.238 \pm 1.119$ & $<0.001$ \\
D23 & $6.892 \pm 2.892$ & $2.345 \pm 1.178$ & $<0.001$ \\
D25 & $7.558 \pm 3.107$ & $2.565 \pm 1.282$ & $<0.001$ \\
D27 & $8.291 \pm 3.363$ & $2.762 \pm 1.334$ & $<0.001$ \\
D29 & $8.985 \pm 3.772$ & $2.929 \pm 1.389$ & $<0.001$ \\
\hline
\end{tabular}

Data are presented as mean $\pm \mathrm{SD}$.

Table III. Comparison of apparent diffusion coefficient values between experimental and control groups at different timepoints after radiotherapy.

\begin{tabular}{lllr}
\hline & $\begin{array}{c}\text { Control group } \\
\left(\times 10^{-6} \mathrm{~mm}^{2} / \mathrm{sec}\right)\end{array}$ & $\begin{array}{c}\text { Experimental group } \\
\left(\mathrm{x} 10^{-6} \mathrm{~mm}^{2} / \mathrm{sec}\right)\end{array}$ & P-value \\
\hline Day & $507.62 \pm 112.62$ & $492.40 \pm 113.22$ & 0.679 \\
D1 & $457.48 \pm 49.35$ & $462.30 \pm 131.71$ & 0.872 \\
D3 & $445.60 \pm 73.99$ & $594.55 \pm 120.87$ & $<0.001$ \\
D5 & $423.70 \pm 61.78$ & $615.38 \pm 84.90$ & $<0.001$ \\
D7 & $414.93 \pm 44.01$ & $645.40 \pm 87.15$ & $<0.001$ \\
D9 & $401.39 \pm 42.89$ & $641.96 \pm 103.28$ & $<0.001$ \\
D11 & $428.90 \pm 68.18$ & $637.12 \pm 86.04$ & $<0.001$ \\
D13 & $420.08 \pm 76.40$ & $607.30 \pm 73.64$ & $<0.001$ \\
D15 & $417.56 \pm 49.46$ & $590.13 \pm 98.61$ & 0.002 \\
D17 & $400.43 \pm 44.08$ & $506.33 \pm 59.38$ & $<0.001$ \\
D19 & $419.90 \pm 57.24$ & $486.75 \pm 83.14$ & 0.008 \\
D21 & $431.51 \pm 88.76$ & $489.71 \pm 57.26$ & 0.029 \\
D23 & $398.40 \pm 94.66$ & $472.25 \pm 80.52$ & 0.012 \\
D25 & $417.09 \pm 73.82$ & $501.53 \pm 116.47$ & 0.014 \\
D27 & $429.28 \pm 57.32$ & $500.05 \pm 123.73$ & 0.039 \\
D29 & $432.28 \pm 60.14$ & $503.39 \pm 125.47$ & 0.022 \\
& & &
\end{tabular}

Data are presented as the mean $\pm \mathrm{SD}$.

day after treatment reaching its lowest point on the 9th day; after which it gradually increased to the pretreatment levels by the 17 th day, where it remained stable at a slightly higher level than before treatment. In the control group, MK began
Table IV. Comparison of mean kurtosis values between experimental and control groups at different timepoints after radiotherapy.

\begin{tabular}{lccr}
\hline & $\begin{array}{c}\text { Control group } \\
\left(\mathrm{x} 10^{-3}\right)\end{array}$ & $\begin{array}{c}\text { Experimental group } \\
\left(\mathrm{x} 10^{-3}\right)\end{array}$ & P-value \\
\hline Day & $782.71 \pm 166.49$ & $799.75 \pm 171.13$ & 0.757 \\
D0 & $821.02 \pm 153.29$ & $712.97 \pm 181.78$ & 0.058 \\
D1 & $867.54 \pm 134.18$ & $640.58 \pm 165.65$ & $<0.001$ \\
D5 & $911.27 \pm 115.73$ & $628.38 \pm 182.32$ & $<0.001$ \\
D7 & $936.58 \pm 193.56$ & $621.72 \pm 92.30$ & $<0.001$ \\
D9 & $951.49 \pm 117.93$ & $602.74 \pm 114.56$ & $<0.001$ \\
D11 & $964.10 \pm 140.67$ & $613.91 \pm 129.21$ & $<0.001$ \\
D13 & $1005.52 \pm 125.77$ & $643.35 \pm 138.54$ & $<0.001$ \\
D15 & $986.63 \pm 151.90$ & $745.79 \pm 117.50$ & $<0.001$ \\
D17 & $1022.76 \pm 135.83$ & $807.09 \pm 147.13$ & $<0.001$ \\
D19 & $955.91 \pm 143.92$ & $843.19 \pm 137.63$ & 0.017 \\
D21 & $1000.91 \pm 103.94$ & $848.89 \pm 126.35$ & $<0.001$ \\
D23 & $1024.61 \pm 163.18$ & $854.71 \pm 190.74$ & 0.006 \\
D25 & $963.46 \pm 131.69$ & $827.65 \pm 189.73$ & 0.017 \\
D27 & $962.62 \pm 152.73$ & $832.95 \pm 182.90$ & 0.025 \\
D29 & $966.81 \pm 158.50$ & $821.24 \pm 180.10$ & 0.012 \\
\hline
\end{tabular}

Data are presented as the mean \pm SD.

to rise gradually on the 1 st day and remained at a relatively high level on the 17th day. There were significant differences in $\mathrm{MK}$ in the experimental group at different time points $(\mathrm{F}=11.837 ; \mathrm{P}<0.001)$, and the control group at different time points $(\mathrm{F}=3.963 ; \mathrm{P}=0.000)$. There was no significant difference between $\mathrm{MK}_{0}$ and $\mathrm{MK}_{1}$ between the two groups, and the MK of the experimental group was lower compared with the control group $(\mathrm{P}<0.05)$ from the 3 rd day after radiotherapy (Table IV).

Comparison of $M D$ before and after radiotherapy. The trend in change in MD was similar to that of the ADC. The experimental group exhibited a small decrease in MD on the 1st day after radiotherapy which increased rapidly on the 3rd day, peaking on the 7 th day, and then gradually decreasing to a relatively stable level on the 17th day, but lower compared with before treatment. The MD of the control group gradually decreased on the first day, reaching its lowest point on the 7th day, after which it increased, but did not reach pretreatment levels. There were differences in MD in the experimental group at different time points $(\mathrm{F}=4.948 ; \mathrm{P}<0.001)$, and there were significant differences in the MD in the control group at different time points $(\mathrm{F}=2.371 ; \mathrm{P}=0.044)$. There was no significant difference between $\mathrm{MD}_{0}$ and $\mathrm{MD}_{1}$ between the two groups, and the MD of the experimental group was higher compared with the control group $(\mathrm{P}<0.05)$ from the 3rd day after radiotherapy (Table V).

$m R N A$ expression of AQP3 in tumors. RT-qPCR showed high expression of AQP3 mRNA in the tumors, and no expression of the other AQPs (Figs. 3 and 4). The relative expression of AQP3 mRNA at matched time points were $1.00 \pm 0.00,0.70 \pm 0.28$, $0.39 \pm 0.27,0.24 \pm 0.15,0.67 \pm 0.31,0.63 \pm 0.21$ and $0.60 \pm 0.25$, 
Table V. Comparison of mean diffusivity values between experimental and control groups at different timepoints.

\begin{tabular}{llcr}
\hline Day & $\begin{array}{c}\text { Control group } \\
\left(\times 10^{-6} \mathrm{~mm}^{2} / \mathrm{sec}\right)\end{array}$ & $\begin{array}{c}\text { Experimental group } \\
\left(\times 10^{-6} \mathrm{~mm}^{2} / \mathrm{sec}\right)\end{array}$ & P-value \\
\hline D0 & $753.21 \pm 232.37$ & $753.54 \pm 227.88$ & 0.996 \\
D1 & $663.96 \pm 136.91$ & $739.67 \pm 197.88$ & 0.191 \\
D3 & $618.95 \pm 76.17$ & $837.14 \pm 196.76$ & $<0.001$ \\
D5 & $600.52 \pm 87.40$ & $912.44 \pm 182.04$ & $<0.001$ \\
D7 & $570.78 \pm 74.43$ & $995.20 \pm 235.50$ & $<0.001$ \\
D9 & $641.01 \pm 115.88$ & $958.93 \pm 226.07$ & $<0.001$ \\
D11 & $694.86 \pm 153.79$ & $922.07 \pm 149.15$ & $<0.001$ \\
D13 & $692.80 \pm 186.12$ & $944.92 \pm 158.75$ & $<0.001$ \\
D15 & $702.47 \pm 137.78$ & $918.65 \pm 143.01$ & $<0.001$ \\
D17 & $663.11 \pm 116.75$ & $853.86 \pm 150.38$ & $<0.001$ \\
D19 & $708.50 \pm 163.64$ & $819.54 \pm 128.34$ & 0.021 \\
D21 & $691.72 \pm 175.65$ & $796.07 \pm 101.64$ & 0.043 \\
D23 & $662.61 \pm 181.83$ & $780.09 \pm 131.66$ & 0.023 \\
D25 & $667.06 \pm 146.94$ & $789.66 \pm 196.80$ & 0.040 \\
D27 & $705.00 \pm 115.20$ & $827.84 \pm 210.91$ & 0.040 \\
D29 & $649.42 \pm 79.02$ & $822.15 \pm 223.47$ & 0.002 \\
\hline
\end{tabular}

Data are presented as the mean $\pm \mathrm{SD}$.

Table VI. Relative expression of aquaporin 3 protein between experimental and control groups.

\begin{tabular}{lccc}
\hline Day & $\begin{array}{c}\text { Experimental } \\
\text { group (\%) }\end{array}$ & $\begin{array}{c}\text { Control } \\
\text { group (\%) }\end{array}$ & P-value \\
\hline D0 & $0.30 \pm 0.06$ & $0.30 \pm 0.06$ & 0.868 \\
D1 & $0.30 \pm 0.10$ & $0.34 \pm 0.13$ & 0.337 \\
D3 & $0.28 \pm 0.03$ & $0.35 \pm 0.06$ & 0.016 \\
D5 & $0.26 \pm 0.07$ & $0.37 \pm 0.08$ & 0.025 \\
D7 & $0.23 \pm 0.08$ & $0.36 \pm 0.08$ & 0.019 \\
D17 & $0.24 \pm 0.03$ & $0.31 \pm 0.03$ & 0.007 \\
D29 & $0.26 \pm 0.02$ & $0.28 \pm 0.03$ & 0.228 \\
\hline
\end{tabular}

respectively. The expression of the AQP3 mRNA before radiotherapy was similar, and the relative expression of AQP3 mRNA began to decrease on the first day after radiotherapy, reaching its lowest point on the 5th day, after which the expression began to increase to a stable level. There were statistically significant differences in the relative expression of AQP3 mRNA at different time points $(\mathrm{F}=5.114$; $\mathrm{P}=0.001$; Fig. 3$)$.

AQP3 protein expression in tumors. The relative expression of AQP3 protein between the experimental group and control group at different time points are shown in Table VI and Fig. 4. Western blotting showed the relative expression of AQP3 protein in the experimental group increased slightly on the first day after radiotherapy, and began to decrease on the third day, reaching its lowest level on the 7th day, after which expression gradually increased. The relative expression of AQP3 protein
Table VII. Comparison of necrosis ratio between experimental and control groups.

\begin{tabular}{lccr}
\hline Day & $\begin{array}{c}\text { Experimental } \\
\text { group (\%) }\end{array}$ & $\begin{array}{c}\text { Control } \\
\text { group (\%) }\end{array}$ & P-value \\
\hline D0 & $30.73 \pm 4.86$ & $32.86 \pm 1.36$ & 0.342 \\
D1 & $31.54 \pm 4.41$ & $31.95 \pm 4.78$ & 0.879 \\
D3 & $32.19 \pm 1.21$ & $29.16 \pm 2.16$ & 0.013 \\
D5 & $38.70 \pm 9.41$ & $26.85 \pm 2.50$ & 0.026 \\
D7 & $53.17 \pm 2.88$ & $29.16 \pm 2.16$ & $<0.001$ \\
D17 & $47.96 \pm 4.45$ & $29.49 \pm 2.17$ & $<0.001$ \\
D29 & $44.06 \pm 2.26$ & $29.49 \pm 2.03$ & $<0.001$ \\
\hline
\end{tabular}

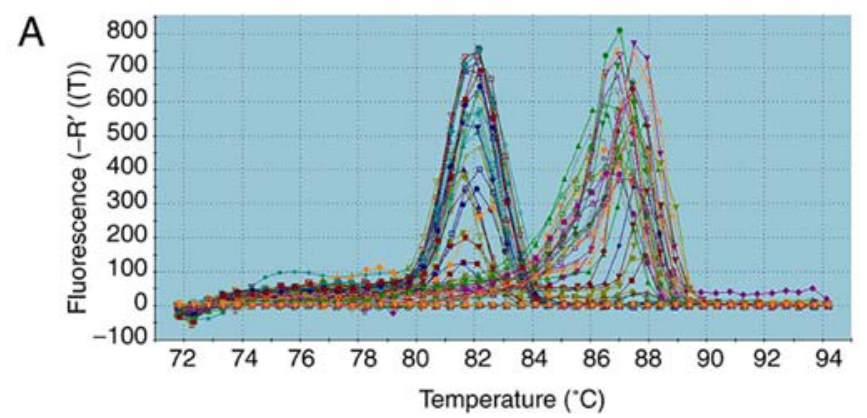

B
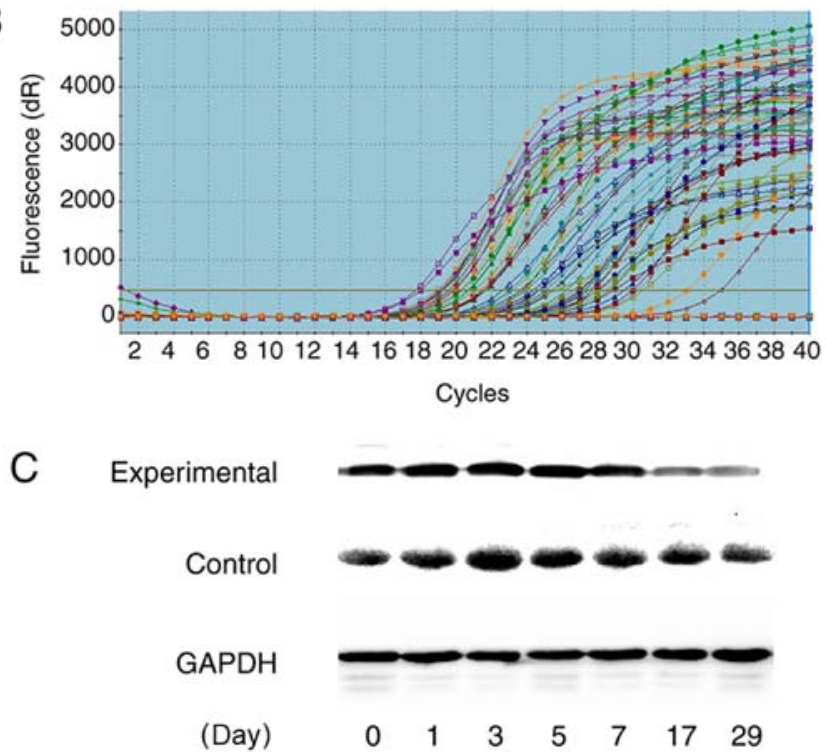

Figure 3. Expression of AQP3. (A) Solubility curve and (B) amplification curve of AQP3 based on quantitative PCR. (C) AQP3 protein expression. GAPDH was used as the loading control. AQP, aquaporin.

in the control group began to increase on the first day after radiotherapy, peaking on the 5th day, and then showing a downward trend in expression. The relative expression of the two groups of AQP3 protein showed statistically significant differences on the 3 rd $(\mathrm{P}=0.016), 5$ th $(\mathrm{P}=0.025)$, 7th $(\mathrm{P}=0.019)$ and 17th (0.007) days after radiotherapy (Fig. 3 and Table VI).

Comparison of the necrosis ratio. The results of necrosis analysis are shown in Table VII. The rate of necrosis in the experimental group gradually increased, but on the 7 th day 
A

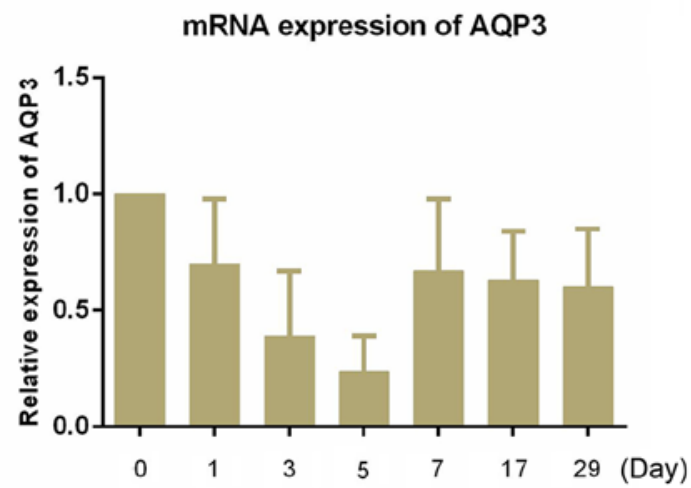

B

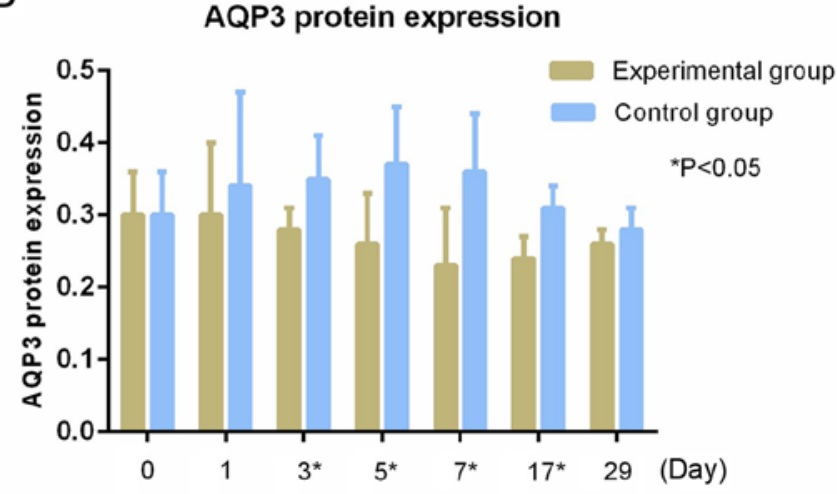

Figure 4. Expression levels of AQP3. (A) mRNA expression of AQP3 in tumors (B) AQP3 protein expression in tumors. AQP, aquaporin.

A

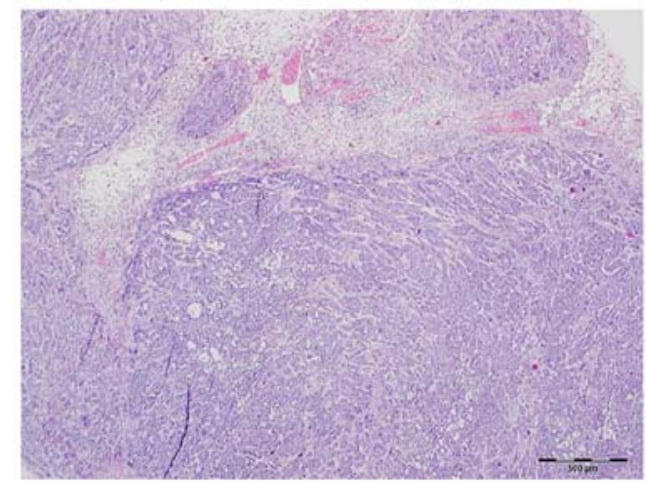

B

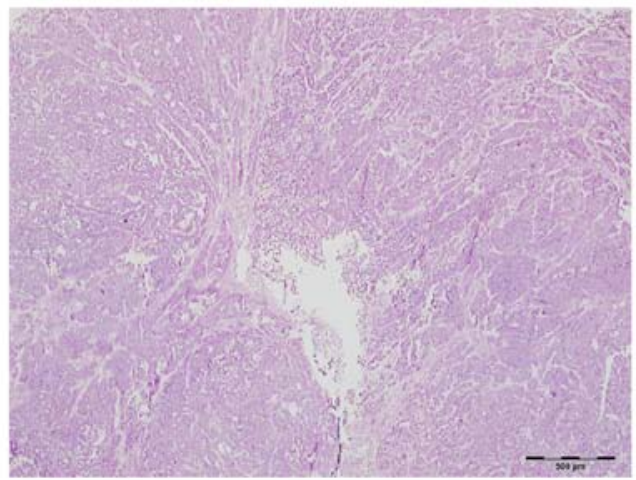

Figure 5. Image of necrosis ratio. Necrosis ratio of transplanted tumors on the 7th day in the (A) experimental group and (B) control group. Necrosis was assessed using hematoxylin and eosin staining. Magnification, x40. Scale bar, $500 \mu \mathrm{m}$.

began to gradually decrease. The control group showed a slightly gradual downward trend and stabilized after the 7 th day. The proportion of necrotic tissue in the experimental group was higher than that of the control group after the 3rd day $(\mathrm{P}<0.05$; Fig. 5).

\section{Discussion}

The ADC, as a quantitative parameter of MRDWI, has shown advantages in evaluating treatment efficacy (23). Quantitative determination of the ADC before, during and/or after chemotherapy and/or radiotherapy is expected to predict treatment response and prognostic assessment (24-28). However, as the $b$ value increases, the diffusion of water molecules within the tissue shows greater deviation from the Gaussian distribution $(4,9)$. However, DKI is based on a non-Gaussian distribution model (29), which may provide more information that is also more accurate (9). In the present study, representative MK and MD values from the animal model were selected to create a new sensitivity index for evaluation of the efficacy of esophageal cancer radiotherapy and provide a theoretical basis for the clinical application of this technique.

The results showed that irradiation with a single dose at 15 Gy did not cause death in nude mice, but it was an effective and feasible dose for observing the change in tumor volume. There was a significant growth delay in human esophageal carcinoma implanted in nude mice with single high-dose radiotherapy. The tumor size in the experimental group grew slowly, and the volume increased at only one time point at the end of the observations, whereas in the control group, tumors grew stably and increased to $\sim 8$ times the initial volume. Tumor growth in the experimental group began to slow starting from the 5th day following radiotherapy, and there was a slight drop on the 7 th day. A possible reason for the increase in volume is tissue edema, and another reason may be due to a secondary effect following a single dose of radiation. Cells can perform several mitotic cycles before death, which is defined as compensatory cell proliferation (30). Subsequently, due to the gradual disappearance of the compensatory proliferative effect of tumor cells, apoptotic signaling pathways were activated by radiotherapy, and tumor cells underwent apoptosis (30), thus tumor growth slowed down or even shrunk. However, a single dose of $15 \mathrm{~Gy}$ radiotherapy was not sufficient to kill all the tumor cells, and the tumor volume in the experimental group continued to increase slowly due to the proliferation of the cells that survived the treatment. In the initial stage, following high-energy X-ray exposure, the cell membranes of tumor tissues were damaged, and this may have led to the dysfunction of active transmembrane transportation of water molecules (31). However, the water molecules from the extracellular regions could still pass through the cell membrane via passive osmosis, thereby resulting in an increase in the intracellular water content and cell density, causing tumor cell edema. The cell interstitial gaps were smaller than before 
radiotherapy, thus the ADC values of the experimental group decreased on the 1st day. Therefore, high dose of radiation may lead to the initiation of apoptosis in xenograft cells (30). The decreased number of tumor cells was likely the result of a lower cell density followed by an enlargement of extracellular space. The restriction of movement of water molecules was relatively eased prior to treatment, and the ADC value increased gradually and reached its peak when the tumor volume was about to decrease (on the 5th day), indicating a substantial decrease in restriction of water diffusion within the extracellular space, intercellular space, or both. With the proliferation of tumors in the control group, the volume gradually increased, and the ADC value gradually decreased. The significant differences in the volumes of the two groups of tumors was observed on the 7th day, whereas the differences in the ADC values were observed on the 3rd day. Changes in the ADC values preceded the change in morphological changes of the tumor. Thus, the observed variation in ADC in the present study appeared to be in line with the expected effect of successful treatment.

The ADC, MD and MK values are all related to the diffusion of water molecules. The MD value is the average of the degree of diffusion of the water molecules in the diffusion gradient field, and it can reflect the overall diffusion, independent of the direction (32). MK is an index which measures the complexity of the organizational structure. The more complex the imaging of the carcinoma structure, the greater the degree of deviation of water molecules from the Gaussian distribution, and the greater the average kurtosis value $(33,34)$. The degree of water molecule diffusion is related to the complexity of the tissue structure; the more notable the difference between tumor tissue and normal tissue and the greater diversity of cell nucleomorphology, the richer the interstitial blood vessels, the greater the cell density, the smaller the extracellular gap, the more limited the diffusion of water molecules and the more complex the structure. This will all result in more deviation of water diffusion from Gaussian distribution and, therefore, smaller ADC and MD values and a larger MK value $(1,2,33,34)$. In the present study, the trends of the MK value were opposite to that of the ADC and MD values. Similar results were also observed following irradiation of nasopharyngeal carcinoma in a xenograft model (35). Induction of MD and reduction of MK were observed during the initial stages of fraction irradiation in xenografts of high radio-sensitive cell-lines, which occurred prior to morphological changes. Therefore, both MD and MK values are valuable imaging markers for the early detection of radio-sensitivity in a xenograft model of nasopharyngeal carcinoma. A previous study showed that measurement of $\mathrm{ADC}$ and MD after chemoradiotherapy (CRT) can reliably differentiate the pathologic complete remission (pCR) from the non-pCR group, and both the ADC and MD values in patients with rectal cancer increased following neoadjuvant chemoradiation therapy, whereas the opposite trend was seen with MK (36). The change ratio of ADC and MD were significantly higher for the $\mathrm{pCR}$ patients than for the non-pCR patients, and the MK showed relatively high sensitivity (92.9\%) and high specificity (83.3\%) in comparison to other imaging indices. The results of the present study were consistent with the above study; tumor growth corresponded to the
ADC, MD and MK values, and the changes in indicators all occurred prior to changes in general tumor morphology. In the present study, the ADC value was based on the reference, which suggests that DKI exhibits predictive value for the sensitivity of esophageal cancer to radiotherapy during the early stages.

AQPs serve a crucial role in tumor growth and are involved in cell migration, proliferation and angiogenesis (37-39). AQPs are strongly expressed in tumor cells of different origins. Kusayama et al (40) reported that AQP3 expression was upregulated in esophageal cancer, and the proliferative and metastatic potential of esophageal squamous cell carcinoma were correlated with AQP3 expression. The correlation between the level of AQP expression in tumor cells and the amount of tumor edema, as revealed by MRI, suggested the involvement of AQPs in tumor edema formation (41). In the present study, AQP3 expression in the experimental group decreased from day 3 onwards, reaching its lowest point on day 7 , and this may be associated with radiotherapy-mediated destruction of cell membranes (31). AQP3 expression in the control group began to increase from the 1st day, reaching its peak on the 5th day, which indicated proliferation and invasion of the tumor (8). AQP3 in the control group then showed a downward trend, which may be associated with tumor necrosis. Furthermore, studies have shown that the ADC value is slightly negatively correlated with AQP3 expression (42-44); however, the association between AQP3 and DKI parameters requires further study.

In the second part of the experiments, the control of tumor growth over time was assessed. The proportion of necrotic cells in the tumor tissues increased following radiotherapy in the experimental group, and this was associated with cell dissolution and tissue necrosis caused by irradiation. However, the necrotic ratio decreased due to tumor cell proliferation and regrouping at the later stages. The control group showed a gradual downward trend in necrotic ratio and tended to stabilize after the 7th day. This may have been due to the rapid proliferation during the early stages and stabilization of proliferation in the later stages of tumor development. The ratio of necrotic cells was significantly higher in the experimental group from day 3 onwards. Similar results were obtained in other animal studies $(45,46)$. Zhong et al (45) found that DKI findings can provide valuable bio-information for nasopharyngeal carcinoma (NPC) tissue characterization. DKI imaging might be utilized as a surrogate biomarker for the non-invasive assessment of tumor microstructures. Zheng et al (46) suggested that Changes in MD and MK parameters after fractional irradiation are closely related with cellular and pathological characteristics, especially size reduction and necrosis induction. These parameters exhibit potential abilities of monitoring the response to fractional irradiation in radio-sensitive NPC xenografts. Both ADC and MD increased with persistent cell death or tumor necrosis following treatment (47), suggesting that these two parameters may be used to reflect the degree of necrosis following treatment. Guo et al (48) found that MD and ADC values of hepatocellular carcinoma in the sorafenib induction group were significantly higher compared with the control group. MD was significantly increased, which may be due to decreased tumor cell density, increased intratumoral necrosis and increased extracellular space. These values again 
showed positive correlation with the histopathological necrotic fraction, and changes in the MK values were observed before morphological changes in hepatocellular carcinoma.

In summary, single high-dose radiotherapy can inhibit tumor growth following radiotherapy, and the ADC, MK and MD values of tumors following radiotherapy were preceded by the change in tumor withdrawal before morphological changes. The pathological comparison showed that the changes in the cell density and necrotic ratio of tumors were in agreement with the change in the ADC, MK and MD values. The present study used several time points to assess the changes, allowing for increased confidence in the changes observed. However, the motion artifacts in some images, as well as the magnetic-sensitive artifacts, which may have occurred when the fit of mice when wrapped in pork was poor, may have affected the accuracy of the data to some extent. Additionally, there appears to be a lack of consensus in b-values $(49,50)$. The stability and reliability of DKI requires additional research and improvements, in addition to clinical studies to further confirm the results of the present study. Additionally, the association between AQP3 and DKI parameters requires further study.

In conclusion, single large fraction doses of radiotherapy can inhibit the growth of a xenograft. ADC, MK and MD were altered prior to morphological changes in the tumor. The change in AQP3 expression and necrosis ratio were associated with DKI parameters. Thus, DKI exhibits early predictive ability for detecting the sensitivity of radiotherapy in esophageal carcinoma.

\section{Acknowledgements}

Thank is given to Dr Shi Qinglei (Siemens) for the technical guidance given for this study.

\section{Funding}

This research is supported by the Public Health Department of Hebei (grant no. 20170170).

\section{Availability of data and materials}

The datasets used and/or analyzed during the present study are available from the corresponding author on reasonable request.

\section{Authors' contributions}

ADZ made substantial contributions to conception and design the study and wrote the manuscript. XHS made substantial contributions to the acquisition of the data and analysis and interpretation of data and involved in drafting the manuscript. YFW and NZ performed the experiments. GFS made substantial contributions to conception of the study, revised the draft critically for important intellectual content and give final approval of the version to be published and agreed to be accountable for all aspects of the work in ensuring that questions related to the accuracy or integrity of any part of the work are appropriately investigated and resolved. $\mathrm{CH}$ made substantial contributions to conception and design, and was responsible for guiding experiments. All authors read and approved the final manuscript.

\section{Ethics approval and consent to participate}

The animal use protocol listed below has been reviewed and approved by the Laboratory Animal Ethical Committee Fourth Hospital Hebei Medical University (approval no. 201618) and complied with institutional, national or international guidelines and relevant guidelines.

\section{Patient consent for publication}

Not applicable.

\section{Competing interests}

The authors declare that they have no competing interests.

\section{References}

1. Patterson DM, Padhani AR and Collins DJ: Technology insight: Water diffusion MRI-a potential new biomarker of response to cancer therapy. Nat Clin Pract Oncol 5: 220-233, 2008.

2. Lambregts DM, Vandecaveye V, Barbaro B, Bakers FC, Lambrecht M, Maas M, Haustermans K, Valentini V, Beets GL and Beets-Tan RG: Diffusion-weighted MRI for selection of complete responders after chemoradiation for locally advanced rectal cancer: A multicenter study. Ann Surg Oncol 18: 2224-2231, 2011.

3. Aoyagi T, Shuto K, Okazumi S, Shimada H, Kazama T and Matsubara H: Apparent diffusion coefficient values measured by diffusionweighted imaging predict chemoradiotherapeutic effect for advanced esophageal cancer. Dig Surg 28: 252-257, 2011.

4. Jensen JH and Helpern JA: MRI quantification of non-Gaussian water diffusion by kurtosis analysis. NMR Biomed 23: 698-710, 2010.

5. Sun K, Chen X, Chai W, Fei X, Fu C, Yan X, Zhan Y, Chen K, Shen K and Yan F: Breast cancer: Diffusion kurtosis MR imaging-diagnostic accuracy and correlation with clinicalpathologic factors. Radiology 277: 46-55, 2015.

6. Agre P, Sasaki S and Chrispeels MJ: Aquaporins: A family of water channel proteins. Am J Physiol 265: F461, 1993.

7. King LS and Agre P: Pathophysiology of the aquaporin water channels. Annu Rev Physiol 58: 619-648, 1996.

8. Verkman AS: More than just water channels: Unexpected cellular roles of aquaporins. J Cell Sci 118: 3225-3232, 2005.

9. Jensen JH, Helpern JA, Ramani A, Lu H and Kaczynski K: Diffusional kurtosis imaging: The quantification of non-gaussian water diffusion by means of magnetic resonance imaging. Magn Reson Med 53: 1432-1440, 2005.

10. Suo S, Chen X, Wu L, Zhang X, Yao Q, Fan Y, Wang H and $\mathrm{Xu} \mathrm{J}$ : Non-Gaussian water diffusion kurtosis imaging of prostate cancer. Magn Reson Imaging 32: 421-427, 2014.

11. Nogueira L, Brandão S, Matos E, Nunes RG, Loureiro J, Ramos I and Ferreira HA: Application of the diffusion kurtosis model for the study of breast lesions. Eur Radiol 24: 1197-1203, 2014.

12. Rosenkrantz AB, Sigmund EE, Winnick A, Niver BE, Spieler B, Morgan GR and Hajdu CH: Assessment of hepatocellular carcinoma using apparent diffusion coefficient and diffusion kurtosis indices: Preliminary experience in fresh liver explants. Magn Reson Imaging 30: 1534-1540, 2012.

13. Van Cauter S, Veraart J, Sijbers J, Peeters RR, Himmelreich U, De Keyzer F, Van Gool SW, Van Calenbergh F, De VleeschouwerS, Van Hecke W and Sunaert S: Gliomas: Diffusion kurtosis MR imaging in grading. Radiology 263: 492-501, 2012.

14. Raab P, Hattingen E, Franz K, Zanella FE and Lanfermann H: Cerebral gliomas: Diffusional kurtosis imaging analysis of microstructural differences. Radiology 254: 876-881, 2010.

15. Rosenkrantz AB, Sigmund EE, Johnson G, Babb JS, Mussi TC, Melamed J, Taneja SS, Lee VS and Jensen JH: Prostate cancer: Feasibility and preliminary experience of a diffusional kurtosis model for detection and assessment of aggressiveness of peripheral zone cancer. Radiology 264: 126-135, 2012. 
16. Fusco R, Petrillo M, Granata V, Filice S, Sansone M, Catalano O and Petrillo A: Magnetic resonance imaging evaluation in neoadjuvant therapy of locally advanced rectal cancer: A systematic review. Radiol Oncol 51: 252-262, 2017.

17. Petrillo M, Fusco R, Catalano O, Sansone M, Avallone A, Delrio P, Pecori B, Tatangelo F and Petrillo A: MRI for assessing response to neoadjuvant therapy in locally advanced rectal cancer using DCE-MR and DW-MR data sets: A preliminary report. Biomed Res Int 2015: 514740, 2015.

18. Pan J, Zang L, Zhang Y, Hong J, Yao Y, Zou C, Zhang L and Chen Y: Early changes in apparent diffusion coefficients predict radiosensitivity of human nasopharyngeal carcinoma xenografts Laryngoscope 122: 839-843, 2012.

19. Yuan ZG, Wang ZY, Xia MY, Li FZ, Li Y, Shen Z and Wang XZ: Comparison of diffusion kurtosis imaging versus diffusion weighted imaging in predicting the recurrence of early stage single nodules of hepatocellular carcinoma treated by radiofrequency ablation. Cancer Imaging 19: 30, 2019.

20. Li T, Hong Y, Kong D and Li K: Histogram analysis of diffusion kurtosis imaging based on whole-volume images of breast lesions. J Magn Reson Imaging 51: 627-634, 2020.

21. Livak KJ and Schmittgen TD: Analysis of relative gene expression data using real-time quantitative PCR and the 2(-Delta Delta C(T)) method. Methods 25: 402-408, 2001

22. Menglin N, Lan W, Chun H, Liang X, Xuejiao R, Shutang L, Liu L and Hua T: Experimental study on radiotherapy response of esophageal cancer xenograft models by diffusion-weighted magnetic resonance imaging method. Chin J Radiol Med Prot 37: 896-901, 2017.

23. Xie H, Sun T, Chen M, Wang H, Zhou X, Zhang Y, Zeng H, Wang $\mathrm{J}$ and $\mathrm{Fu} \mathrm{W}$ : Effectiveness of the apparent diffusion coefficient for predicting the response to chemoradiationtherapy in locally advanced rectal cancer: A systematic review and meta-analysis. Medicine (Baltimore) 94: e517, 2015.

24. Zheng H, Ren W, Pan X, Zhang Q, Liu B, Liu S, He J and Zhou Z: Role of intravoxel incoherent motion MRI in early assessment of the response of esophageal squamous cell carcinoma to chemoradiotherapy: A pilot study. J Magn Reson Imaging 48: 349-358, 2018.

25. Giganti F, Salerno A, Ambrosi A, Chiari D, Orsenigo E, Esposito A, Albarello L, Mazza E, Staudacher C, Del Maschio A and De Cobelli F: Prognostic utility of diffusion weighted MRI in oesophageal cancer: Is apparent diffusion coefficient a potential marker of tumour aggressiveness? Radiol Med 121: 173-180, 2016.

26. Liu S, Zhen F, Sun N, Chen J, Cao Y, Zhang S, Cheng H, Ge X and Sun X: Apparent diffusion coefficient values detected by diffusion-weighted imaging in the prognosis of patients with locally advanced esophageal squamous cell carcinoma receiving chemoradiation. Onco Targets Ther 9: 5791-5796, 2016

27. Wang L, Liu L, Han C, Liu S, Tian H, Li Z, Ren X, Shi G, Wang $Q$ and Wang G: The diffusion-weighted magnetic resonance imaging (DWI) predicts the early response of esophageal squamous cell carcinoma to concurrent chemoradiotherapy. Radiother Oncol 121: 246-251, 2016

28. van Rossum PSN, van Hillegersberg R, Lever FM, Lips IM van Lier AL, Meijer GJ, van Leeuwen MS, van Vulpen M and Ruurda JP: Imaging strategies in the management of oesophageal cancer: What's the role of MRI? Eur Radiol 23: 1753-1765, 2013.

29. Wu D, Li G, Zhang J, Chang S, Hu J and Dai Y: Characterization of breast tumors using diffusion kurtosis imaging (DKI). PLoS One 9: e113240, 2014

30. Ou G, Itasaka S, Zeng L, Shibuya K, Yi J, Harada H and Hiraoka M: Usefulness of HIF-1 imaging for determining optimal timing of combining bevacizumab and radiotherapy. Int J Radiat Oncol Biol Phys 75: 463-467, 2009.

31. Freeman A, Hetzel U, Cripps P and Mobasheri A: Expression of the plasma membrane markers aquaporin 1 (AQP1), glucose transporter 1 (GLUT1) and Na, K-ATPase in canine mammary glands and mammary tumours. Vet J 185: 90-93, 2010.

32. Filli L, Wurnig M, Nanz D, Luechinger R, Kenkel D and Boss A: Whole-body diffusion kurtosis imaging: Initial experience on non-Gaussian diffusion in various organs. Invest Radiol 49: 773-778, 2014

33. Zhu L, Pan Z, Ma Q, Yang W, Shi H, Fu C, Yan X, Du L, Yan F and Zhang H: Diffusion kurtosis imaging study of rectal adenocarcinoma associated with histopathologic prognostic factors: Preliminary findings. Radiology 284: 66-76, 2017.
34. Liu X, Zhou L, Peng W, Wang H and Zhang Y: Comparison of stretched-exponential and monoexponential model diffusion-Weighted imagingin prostate cancer and normal tissues. J Magn Reson Imaging 42: 1078-1085, 2015.

35. Zheng X, Chen Y, Xiao Y, Zheng D and Chen W: Early diagnosis of radio-insensitive human nasopharyngeal carcinoma xenograft models bydiffusion kurtosis imaging. Magn Reson Imaging 55: 128-132, 2019.

36. Hu F, Tang W, Sun Y, Wan D, Cai S, Zhang Z, Grimm R, Yan X, $\mathrm{Fu} \mathrm{C}$, Tong $\mathrm{T}$ and Peng $\mathrm{W}$ : The value of diffusion kurtosis imaging in assessing pathological complete response to neoadjuvant chemoradiation therapy in rectal cancer: A comparison with conventional diffusion-weighted imaging. Oncotarget 8: 75597-75606, 2017.

37. Verkman AS, Hara-Chikuma $M$ and Papadopoulos MC: Aquaporins-new players in cancer biology. J Mol Med (Berl) 86: 523-529, 2008

38. Papadopoulos MC, Saadoun S and Verkman AS: Aquaporins and cell migration. Pflugers Arch 456: 693-700, 2008.

39. $\mathrm{Hu} \mathrm{J}$ and Verkman AS: Increased migration and metastatic potential of tumor cells expressing aquaporin water channels. FASEB J 20: 1892-1894, 2006.

40. Kusayama M, Wada K, Nagata M, Ishimoto S, Takahashi $H$, Yoneda M, Nakajima A, Okura M, Kogo M and Kamisaki Y: Critical role of aquaporin 3 on growth of human esophageal and oral squamous cell carcinoma. Cancer Sci 102: 1128-1136, 2011.

41. Nico B, Mangieri D, Tamma R, Longo V, Annese T, Crivellato E Pollo B, Maderna E, Ribatti D and Salmaggi A: Aquaporin-4 contributes to the resolution of peritumoural brain oedema in human glioblastoma multiforme after combined chemotherapy and radiotherapy. Eur J Cancer 45: 3315-3325, 2009.

42. Tan Y, Zhang H, Wang XC, Qin JB and Wang L: The value of multi ultra high-b-value DWI in grading cerebral astrocytomas and its association with aquaporin-4. Br J Radiol 91: 20170696, 2018.

43. Kong L, Lian G, Zheng W, Liu H, Zhang H and Chen R: Effect of alcohol on diffuse axonal injury in rat brainstem: Diffusion tensor imaging and aquaporin-4 expression study. Biomed Res Int 2013: 798261, 2013.

44. Yao X, Derugin N, Manley GT and Verkman AS: Reduced brain edema and infarct volume in aquaporin-4 deficient mice after transient focal cerebral ischemia. Neurosci Lett 584: 368-372, 2015.

45. Zhong J, Shi P, Chen Y, Huang R, Xiao Y, Zheng X, Zheng D and Peng L: Diffusion kurtosis imaging of a human nasopharyngeal carcinoma xenograft model: Initial experience with pathological correlation. Magn Reson Imaging 47: 111-117, 2018.

46. Zheng X, Chen Y, Zheng D, Xiao Y, Chen J, Pan J and Chen W: Diffusion kurtosis imaging and tumour microstructure for monitoring response to radiotherapy in human nasopharyngeal carcinoma xenografts. Jpn J Clin Oncol 50: 548-555, 2020

47. Yang SH, Lin J, Lu F, Han ZH, Fu CX, Lv P, Liu H and Gao DM: Evaluation of antiangiogenic and antiproliferative effects of sorafenib by sequential histology and intravoxel incoherent motion diffusion-weighted imaging in an orthotopic hepatocellular carcinoma xenograft model. J Magn Reson Imaging 45: 270-280, 2017.

48. Guo R, Yang SH, Lu F, Han ZH, Yan X, Fu CX, Zhao ML and Lin J: Evaluation of intratumoral heterogeneity by using diffusion kurtosis imaging and stretched exponential diffusion-weighted imaging in an orthotopic hepatocellular carcinoma xenograft model. Quant Imaging Med Surg 9: 1566-1578, 2019.

49. Zhu L, Cheng Q, Luo W, Bao L and Guo G: A comparative study of apparent diffusion coefficient and intravoxel incoherent motion-derivedparameters for the characterization of common solid hepatic tumors. Acta Radiol 56: 1411-1418, 2015.

50. Liu G, Yang Z, Li T, Yang L, Zheng X and Cai L: Optimization of b-values in diffusion-weighted imaging for esophageal cancer: Measuring the longitudinal length of gross tumor volume and evaluating chemoradiotherapeutic efficacy. J Cancer Res Ther 13: 748-755, 2017.

This work is licensed under a Creative Commons Attribution-NonCommercial-NoDerivatives 4.0 International (CC BY-NC-ND 4.0) License. 